\title{
Comparison of dosimetric characteristics of physical wedge and enhanced dynamic wedge in inhomogeneous medium using Monte Carlo simulations
}

\author{
Seied Rabi Mahdavi', Atefeh Mahmoudi², Ghazale Geraily², Ahmad Mostaar ${ }^{3}$ Golbarg Esmaili ${ }^{4}$ \\ ${ }^{1}$ Department of Medical Physics, Faculty of Medicine, Iran University of Medical Sciences, Tehran, Islamic Republic of Iran \\ ${ }^{2}$ Department of Medical Physics and Biomedical Engineering, Faculty of Medicine, Tehran University of Medical Sciences, Tehran, \\ Islamic Republic of Iran \\ ${ }^{3}$ Department of Medical Physics and Biomedical Engineering, Faculty of Medicine, Shahid Beheshti University of Medical Sciences, Tehran, \\ Islamic Republic of Iran \\ ${ }^{4}$ Pars Hospital, Tehran, Islamic Republic of Iran
}

\begin{abstract}
Background: Widely used physical wedges in clinical radiotherapy lead to beam intensity attenuation as well as the beam hardening effect, which must be considered. Dynamic wedges devised to overcome the physical wedges (PWs) problems result in dosimetry complications due to jaw movement while the beam is on.

This study was aimed to investigate the usability of physical wedge data instead of enhanced dynamic wedge due to the enhanced dynamic wedge (EDW) dosimetry measurement hardships of Varian 2100CD in inhomogeneous phantom by Monte Carlo code as a reliable method in radiation dosimetry.

Materials and methods: A PW and EDW-equipped-linac head was simulated using BEAMnrc code. DOSXYZnrc was used for three-dimensional dosimetry calculation in the CIRS phantom.

Results: Based on the isodose curves, EDW generated a less scattered as well as lower penumbra width compared to the PW. The depth dose variations of PWs and EDWs were more in soft tissue than the lung tissue. Beam profiles of PW and EDW indicated good coincidence in all points, except for the heel area.

Conclusion: Results demonstrated that it is possible to apply PW data instead of EDW due to the dosimetry and commissioning hardships caused by EDW in inhomogeneous media.

Key words: physical wedge; enhanced dynamic wedge; Monte Carlo code; inhomogeneous medium Rep Pract Oncol Radiother 2021;26(1):59-65
\end{abstract}

\section{Introduction}

In conformal radiotherapy, physical wedges (PWs) made of high-density materials (e.g. steel or lead) are used to change isodose curves in order to achieve more complete target dose conformity while also preserving nearby normal tissues better.
However, the main concern related to PWs is beam intensity attenuation, which results in the beam hardening effect across the beam path, and must be taken into account in treatment planning systems. This problem introduced the idea of using dynamic or virtual wedges [1-4]. Wedge-Shaped dose distribution in dynamic wedges is created by sweeping

Address for correspondence: Ghazale Geraily, Associate Professor, Department of Medical Physics and Biomedical Engineering, Faculty of Medicine, Tehran University of Medical Sciences, Islamic Republic of Iran, tel: +989124308726; e-mail: gh-geraily@sina.tums.ac.ir 
of an independent jaw within the treatment field during irradiation. This design includes considerable advantages compared to physical wedges, such as the absence of beam hardening, out-of-field dose reduction, shorter treatment time, and being automatic and easy to handle [6-8].

The presented dynamic wedges by Varian could offer angles of $15^{\circ}, 30^{\circ}, 45^{\circ}$, and $60^{\circ}$ for symmetrical field sizes of 4 to $20 \mathrm{~cm}$. This limited accessibility to wedge angles and field sizes was overcome by developing enhanced dynamic wedges (EDWs) which produce more wedge angles and also asymmetric field sizes [6].

In the presence of EDWs, the dosimetry measurements required for implementation in treatment planning systems are complicated due to the jaw movement. Monte Carlo simulation as a reliable and accurate method can be used, which is acknowledged in radiotherapy dosimetry $[9,10]$.

Studies considering the difference between physical wedges and enhanced dynamic wedges were confined to homogeneous media, and few studies were conducted to investigate these differences in inhomogeneous media $[4,8-14,15-19]$.

The goal of this study was to compare the dosimetric properties of PWs and EDWs to assess the possibility of applying physical wedge data instead of enhanced dynamic wedge due to the dosimetry and commissioning hardships caused by enhanced dynamic wedges. This study was conducted based on Monte Carlo studies in chest radiotherapy in breast cancer as a technique with the highest usage of wedges.

\section{Materials and methods}

\section{Open field, PW, and EDW-equipped-linac head simulation}

Geometry and radiation transport simulation of the Varian 2100CD for a nominal 6MV photon beam was performed using the BEAMnrc code, the user code of EGSnrc. The EGSnrc is a developed version of the EGS4 code in which transport physics is greatly improved compared to EGS4 [22]. Premade components called module presented by the BEAMnrc code for geometry simulation have made this code different from other simulation codes. For modeling linac head, modules of SLABS, CONS3R, FLATFILT, CHAMBER, MIROR, and JAWS were used to model target, primary collimator, flattening filter, ion chamber, mirror, and secondary collimator, respectively. PW and EDW with wedge angles $\left(15^{\circ}, 30^{\circ}, 45^{\circ}\right.$, and $\left.60^{\circ}\right)$ and $\left(10^{\circ}, 15^{\circ}, 20^{\circ}, 25^{\circ}, 30^{\circ}\right.$, $45^{\circ}$, and $60^{\circ}$ ) were simulated by PYRAMIDS and DJAWS, respectively. To simulate EDW, AUTOJAWS, a MATLAB-based program written by Kakakhel [23] was used. A Gaussian electron pencil beam with $6.1 \mathrm{MeV}$ energy and $2.8 \mathrm{~cm}$ FWHM as the best match was selected $[13,24,25]$. Photon and electron cut-off energies were set to 0.01 and 0.7 $\mathrm{MeV}$, respectively. Moreover, $5 \times 10^{8}$ initial photons were simulated to achieve statistical uncertainties $<1 \%$. To improve uncertainty and enhance simulation speed, directional bremsstrahlung splitting (DBS), a bremsstrahlung splitting technique, was employed as a variance reduction technique [26].

DOSXYZnrc, another EGSnrc user code, was implemented for three-dimensional (3D) dose calculations [27]. To that end, a voxel-based water phantom with voxel sizes of $0.5 \mathrm{~cm}$ and $0.2 \mathrm{~cm}$ in the penumbra region was simulated to obtain PDD and beam profiles. The phase space file produced by BEAMnrc execution was used as a source in the DOSXYZnrc code. To achieve statistical errors < $1 \%, 10^{9}$ histories were allocated. Afterwards, PDD curves and beam profiles at the $1.6,3.5$, and $10 \mathrm{~cm}$ depths, for field sizes of $10 \times 10$ and $15 \times 15 \mathrm{~cm}^{2}$, were calculated through the STATDOSE program by reading the ".3ddose" file for all three simulated modes of wedge free (i.e. open field), PW, and EDW fields.

\section{Measurement}

To obtain PDD curves and beam profiles for open and physical wedged fields with wedge angles of $15^{\circ}, 30^{\circ}, 45^{\circ}$, and $60^{\circ}$, a $0.6 \mathrm{cc}$ Farmer ionization chamber and a $50 \times 50 \times 50 \mathrm{~cm}^{3}$ water phantom were used. PDD and beam profile measurements were performed at SSD $=100 \mathrm{~cm}$ and $1.6,3.5$, and $10 \mathrm{~cm}$ depths, for field sizes of $10 \times 10$ and 15 $\times 15 \mathrm{~cm}^{2}$. For enhanced dynamic wedged fields, calibrated EBT3 films inserted vertically between Perspex phantom layers were used for PDD curve measurement for seven wedge angles, SSD $=100$ $\mathrm{cm}$ and $10 \times 10 \mathrm{~cm}^{2}$ field size. Beam profile measurements were performed using the calibrated SUN NUCLEAR PROFILER2 profiler at depths of 1.6 and $10 \mathrm{~cm}$ in a Perspex phantom, $S S D=100 \mathrm{~cm}$, and field sizes of $10 \times 10$ and $15 \times 15 \mathrm{~cm}^{2}$. Finally, all simulated and measured PDD curves and beam 
profiles were compared to validate the Monte Carlo simulation model.

Then, the CIRS Model 002LFC IMRT Thorax phantom was employed to investigate dose distribution in lung heterogeneity. The 002LFC has an elliptical shape to simulate an average human torso phantom with dimensions of $30 \times 30 \times 20 \mathrm{~cm}^{3}$.

CT scanned slices of the Thorax phantom were inserted into the Coreplan treatment planning system and, then, a treatment plan with tangential beams (medial and lateral) of $6 \mathrm{MV}$ was designed to prescribe a daily dose of $200 \mathrm{cGy}$ to the central point (in the lung tissue) (Fig. 1).

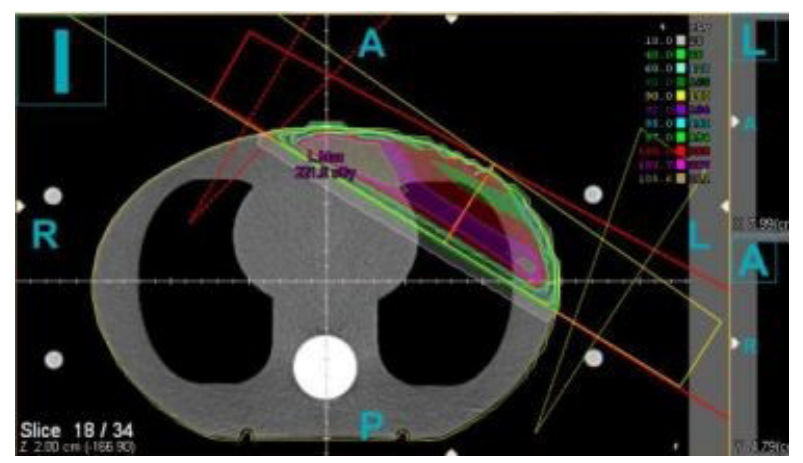

Figure 1. Dose distribution of CIRS inhomogeneous phantom treatment planning

\section{PW and EDW simulations in the inhomogeneous medium}

For dose calculation in the inhomogeneous medium, the CT-based phantom through CTCREATE program was made from CT images of CIRS phantom. The phase space file, created by the BEAMnrc code, was used as a source in DOSXYZnrc code. Moreover, to apply the treatment plan to the CT data in the code and draw isodose curves (Fig. 2), the DOCTP program based on MATLAB program by Dr. Chow was used [28].

\section{Results}

\section{Validation}

To validate the Monte Carlo simulation process, simulated depth dose curves and beam profiles of open, physical, and dynamic wedged fields were compared to those of the measurements. The agreement between simulated and measured profiles of open field was $2 \%$ and $2 \mathrm{~mm}$ for $6.1 \mathrm{MeV}$ energy and $2.8 \mathrm{~cm}$ FWHM. Moreover, the differences between simulated and measured profiles and depth dose curves of PW and EDW were within 2\% and $2 \mathrm{~mm}$ for low- and high-gradient dose regions, respectively.

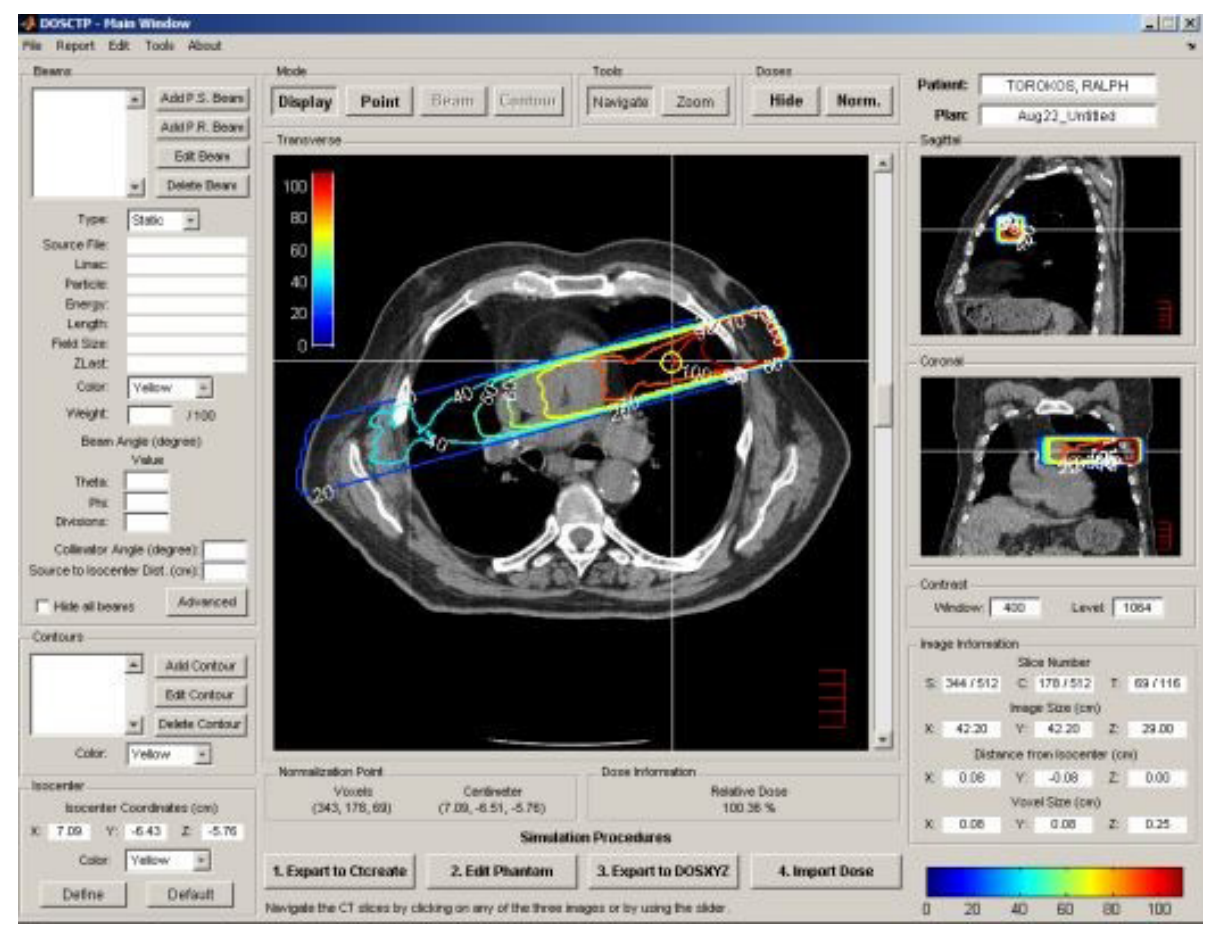

Figure 2. Isodose curves on a CT cut in DOCTP program 


\section{Dosimetric features comparison of PW and EDW in the inhomogeneous medium}

Simulated isodose curves, depth dose curves, and beam profiles parallel and perpendicular to the tangential beams were adopted for the quantitative and qualitative comparison of PW and EDW in the inhomogeneous phantom.

\section{Isodose curve}

Figure 3 illustrates the simulated isodose curves for PW and EDW in the inhomogeneous breast phantom. Evidently, isodose curves become curved out at the lung entrance owing to the lateral electron equilibrium loss.

\section{Depth dose}

Depth dose curves parallel to the tangential beam in the inhomogeneous phantom for PW and EDW are presented in Figure. 4. Table 1 shows the mean and maximum discrepancies of two curves within the soft tissue (in the initial $11 \mathrm{~cm}$, left of the arrow) and in the lung tissue (the next $9 \mathrm{~cm}$, right of the arrow).

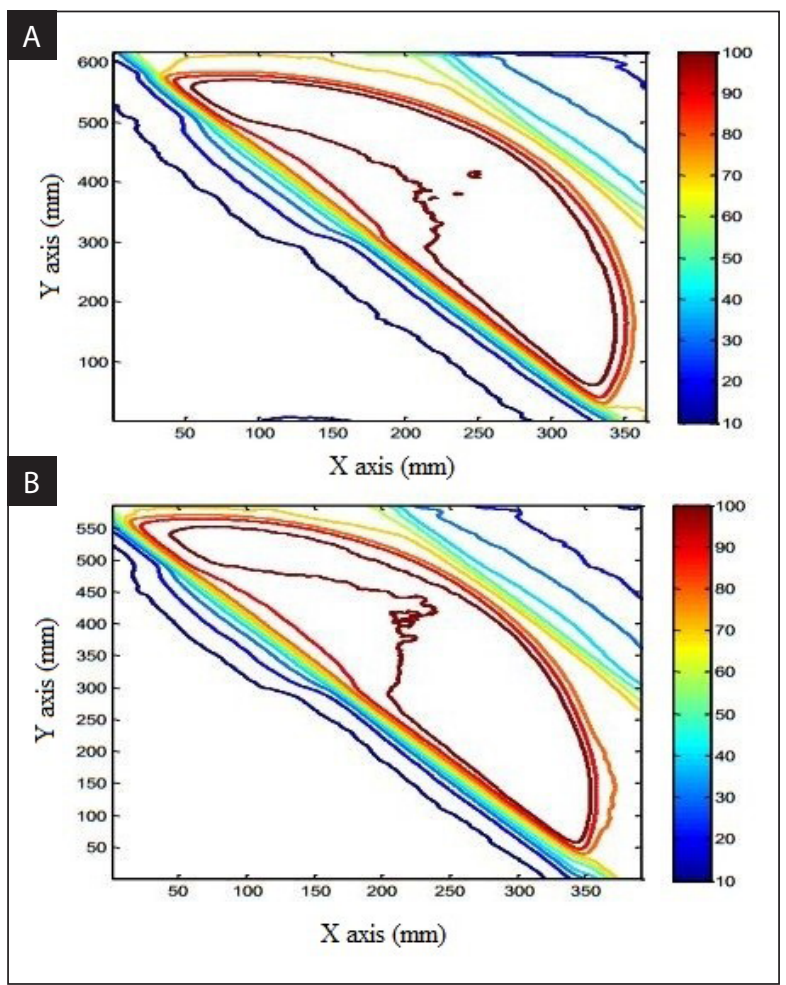

Figure 3. The physical wedge (PW) (A) and the enhanced dynamic wedge (EDW) (B) isodose curves in the inhomogeneous phantom

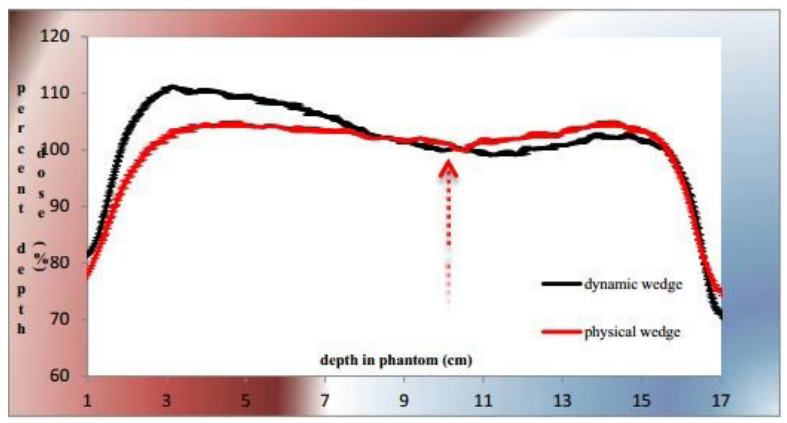

Figure 4. Depth dose variations along the radiation beams passing through the physical wedge (PW) and the enhanced dynamic wedge (EDW) in the inhomogeneous phantom; the arrow indicates the soft tissue-lung interface

Table 1. The mean and maximum differences of the physical wedge (PW) and the enhanced dynamic wedge (EDW) depth dose (DD) curves in soft and lung tissue

\begin{tabular}{|l|c|c|}
\hline \multicolumn{2}{|c|}{$\overline{\mathrm{DD}} \pm$ STD (\%) } & Max DD (\%) \\
\hline $\begin{array}{l}\text { Within the soft tissue } \\
\text { (central area) }\end{array}$ & $-4.27 \pm 0.25$ & 7.92 \\
\hline Within the lung tissue & $2.03 \pm 0.17$ & 2.65 \\
\hline
\end{tabular}

STD — standard deviation

\section{Beam profile}

The beam profile perpendicular to the tangential beam in the inhomogeneous phantom for PW and EDW is depicted in Figure. 5. Two curves differences within various central, toe, and heel areas are presented in Table 2.

\section{Discussion}

In this study, dosimetric characteristics of PW and EDW fields were investigated using Monte Carlo simulation in an inhomogeneous CIRS phantom for the first time. Isodose curves become curved out at the lung entrance because of the lateral electron equilibrium loss due to lower lung density which increases the secondary electron range and scattered photons. As shown in Figure 3 , the curvature of the PW isodose lines near the beam edge is more than the EDW. In other words, the PW penumbra widening is higher compared to the EDW. This can be attributed to the bigger hardening effect produced by PW resulting in increased secondary electron range, which is in accordance with previous studies [21]. On the other hand, EDWs generate less scattered radiation in comparison with PWs which is also reported 


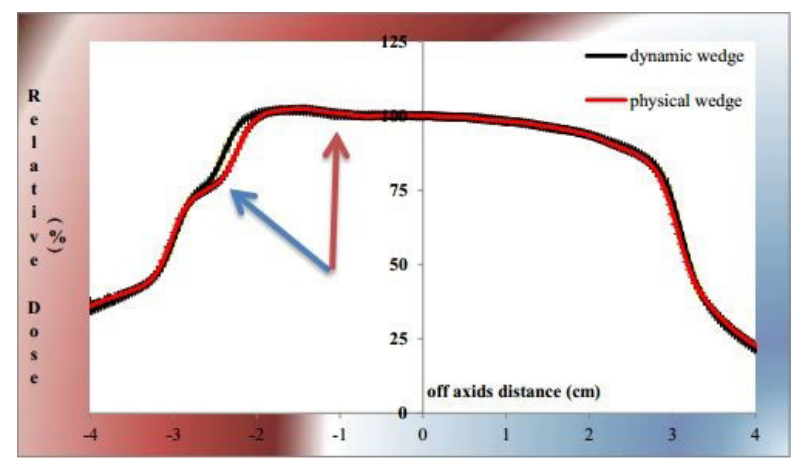

Figure 5. Beam profile variations perpendicular to the radiation beams passing through the physical wedge (PW) and the enhanced dynamic wedge (EDW) in the inhomogeneous phantom; blue and red arrows indicate the air-soft tissue and soft tissue-lung interface, respectively

Table 2. DD and DTA comparative values of PW and EDW profile comparison in the inhomogeneous medium

\begin{tabular}{|c|c|c|}
\hline $\begin{array}{c}\text { Toe area } \\
\overline{\text { DTA }} \pm \text { STD }[\mathrm{mm}]\end{array}$ & $\begin{array}{c}\text { Heel area } \\
\overline{\text { DTA }} \pm \text { STD }[\mathrm{mm}]\end{array}$ & $\begin{array}{c}\text { Central area } \\
\overline{\mathrm{DTA}} \pm \mathrm{STD}[\mathrm{mm}]\end{array}$ \\
\hline $1.24 \pm 0.14$ & $1.95 \pm 0.32$ & $0.1 \pm 0.00$ \\
\hline
\end{tabular}

by Akram et al. [20]. Thus, penumbra widening mainly resulting from scattered photons and secondary electrons is lower for EDW fields. This feature is in agreement with that reported in other studies $[15,29]$.

The 80 and $90 \%$ isodose lines curvature is observed towards the denser tissue due to the diminished backscattered photons arising from the lung tissue. This curvature was observed more prominently for the EDW than the PW. This can be justified by the fact that, with more beam hardening produced by $\mathrm{PW}$, scattered photons are more forward as a result of enhanced energy. Therefore, a greater curvature of the EDW isodose lines was observed resulting from the more lateral scattering.

According to the Figure 4 and Table 1, depth dose variations along the tangential beams, the central axis of the PW and EDW fields is higher in the homogeneous soft tissue compared to the inhomogeneous lung tissue, since the beam central axis passes near the soft tissue-lung interface which leads to increased lateral scattering. Decreased scattering angle of the hardened beam produced by the PW is responsible for less re- ceived dose compared to the EDW. Where the central axis passes through the lung tissue, the role of lateral scattered dose and two curves variations is diminished due to the increased distance from the tissues border.

The physical wedge and EDW beam profiles show good adaption in all points (Fig. 5). The variations in the heel area are a little higher than the central and toe areas (Tab. 2), which is consistent with the previous studies [21].

The blue arrow in Figure 5 represented the airsoft tissue interface. This part corresponds to the wedge heel in the negative area of the $\mathrm{X}$-axis. Due to the electron equilibrium loss in the air and soft tissue intersection, dose reduction is observed. At the entrance to the soft tissue, the EDW absorbed dose is slightly higher than that of PW, because the higher importance of the beam hardening effect in the PW heel area results in more forward scattering of the radiation passing the PW. Thus, less scattered radiation is involved in the absorbed dose. In contrast, the increased scattering angle of enhanced dynamic wedged-beam increases the scattered photon contribution in the absorbed dose near the tissue interfaces.

In the positive area of the X-axis corresponding to the wedge toe and inside the lung tissue, two curves are perfectly matched because of the lower beam hardening effect in the wedge toe. Furthermore, this part of the tissue is placed farther away from the border, so the absorbed dose is chiefly arising from the primary radiation. Despite all the differences between PW and EDW profiles, the two curve discrepancies are negligible. Thus, the PW profiles can be used instead of the EDW. In general, according to the comparisons, it is possible to apply PW dosimetry parameters instead of EDW in the heterogeneous medium.

\section{Conclusion}

In this study, the PW and EDW isodose, depth dose, and profile were compared by Monte Carlo studies in a heterogeneous medium. Quantitative comparison of PW and EDW characteristics in the inhomogeneous medium demonstrated the possibility of applying profiles data of PW instead of EDW. 


\section{Conflict of interest}

None declared.

\section{Funding}

This study was funded and supported by Tehran University of Medical Sciences (TUMS), Tehran, Iran; Grant no. 29995.

\section{References}

1. Saminathan S, Manickam R, Supe SS. Comparison of dosimetric characteristics of physical and enhanced dynamic wedges. Rep Pract Oncol Radiother. 2011; 17(1): 4-12, doi: 10.1016/j.rpor.2011.06.007, indexed in Pubmed: 24376991.

2. Dawod T, Abdelrazek EM, Elnaggar M, et al. Dose Validation of Physical Wedged Asymmetric Fields in Artiste Linear Accelerator. Int J Med Phys Clin Engineer Radiat Oncol. 2014;03(04): 201-209, doi: 10.4236/ijmpcero.2014.34026.

3. Zabihzadeh M, Birgani MJ, Hoseini-Ghahfarokhi M, et al. Dosimetric Characteristics of 6 MV Modified Beams by Physical Wedges of a Siemens Linear Accelerator. Asian Pac J Cancer Prev. 2016; 17(4): 1685-1689, doi: 10.7314/ apjcp.2016.17.4.1685, indexed in Pubmed: 27221838.

4. Shahnawaz A, Subrat SK, Vinod GK, et al. Comprehensive Study of Varian's Enhanced Dynamic Wedge and Physical Wedge. J. Appl Phys. 2017; 9(5): 1-8, doi: 10.9790/48610905040108.

5. Geraily G, Mirzapour M, Mahdavi SR, et al. Monte Carlo study on beam hardening effect of physical wedges. Int J Radiat Res. 2014; 12(3): 249-256.

6. Clinac CS. Enhanced dynamic wedge implementation guide. Varian Oncology Systems 1996.

7. Chang SX, Gibbons JP Clinical implementation of nonphysical wedges. 1999 AAPM Refresher Course. https:// www.aapm.org/meetings/99AM/pdf/2803-83010.pdf.

8. SA SK, P A, J SJ, et al. Comparison of Beam Profiles and Wedge Factors for Physical And Enhanced Dynamic Wedge. Int J Radiol Radiat Ther. 2018; 5(1), doi: 10.15406/ ijrrt.2018.05.00129.

9. Elder PJ, Coveney FM, Welsh AD. An investigation into the comparison between different dosimetric methods of measuring profiles and depth doses for dynamic wedges on a Varian $600 \mathrm{C}$ linear accelerator. Phys Med Biol. 1995; 40(4): 683-689, doi: 10.1088/0031-9155/40/4/013, indexed in Pubmed: 7610122.

10. Verhaegen F, Liu HH. Incorporating dynamic collimator motion in Monte Carlo simulations: an application in modelling a dynamic wedge. Physics in Medicine \& Biology, 46, p. $2001 ; 46(2): 287-296$, doi: 10.1088/0031-9155/46/2/302, indexed in Pubmed: 11229715.

11. Verhaegen F, Das IJ. Monte Carlo modelling of a virtual wedge. Phys Med Biol. 1999; 44(12): N251N259, doi: 10.1088/0031-9155/44/12/402, indexed in Pubmed: 10616157.

12. Shih R, Li XA, Chu JC. Dynamic wedge versus physical wedge: a Monte Carlo study. Med Phys. 2001; 28(4): 612-619, doi: 10.1118/1.1359249, indexed in Pubmed: 11339759.
13. Ahmad M, Deng J, Lund MW, et al. Clinical implementation of enhanced dynamic wedges into the Pinnacle treatment planning system: Monte Carlo validation and patient-specific QA. Phys Med Biol. 2009; 54(2): 447-465, doi: 10.1088/0031-9155/54/2/018, indexed in Pubmed: 19098353.

14. Kakakhel MB, Baveas ES, Fielding AL, et al. Validation and automation of the DYNJAWS component module of the BEAMnrc Monte Carlo code. Australas Phys Eng Sci Med. 2011; 34(1): 83-90, doi: 10.1007/s13246-011-0060-x, indexed in Pubmed: 21424376.

15. Bidmead AM, Garton AJ, Childs PJ. Beam data measurements for dynamic wedges on Varian 600C (6 MV) and $2100 \mathrm{C}$ ( 6 and $10 \mathrm{MV}$ ) linear accelerators. Phys Med Biol. 1995; 40(3): 393-411, doi: 10.1088/0031-9155/40/3/005, indexed in Pubmed: 7732070.

16. Lee JW, Hong S, Choi KS, et al. Comparison of Enhanced Dynamic Wedge with Physical Metal Wedge based on the Basic Dosimetric Parameters. Korean J Med Phys. 2005; 16: 70-75.

17. Ahmad M, Hussain A, Muhammad W, et al. Studying wedge factors and beam profiles for physical and enhanced dynamic wedges. J Med Phys. 2010; 35(1): 33-41, doi: 10.4103/0971-6203.57116, indexed in Pubmed: 20177568.

18. Lahooti A, Takavar A, Nedaei HA. 1101 poster Comparision of surface and peripheral radiotherapy dose using enhanved dynamic and physical wedges. Radiotherapy and Oncology. 2011; 99: S410, doi: 10.1016/s01678140(11)71223-3.

19. Saminathan S, Manickam R, Supe SS. Comparison of dosimetric characteristics of physical and enhanced dynamic wedges. Rep Pract Oncol Radiother. 2011; 17(1): 4-12, doi: 10.1016/j.rpor.2011.06.007, indexed in Pubmed: 24376991.

20. Akram $M$, Iqbal $K$, Isa $M$, et al. Optimum reckoning of contra lateral breast dose using physical wedge and enhanced dynamic wedge in radiotherapy treatment planning system. Int J Radiat Res. 2014; 12(4): 295-302.

21. Geraily G, Sharafi N, Shirazi A, et al. Comparison of beam hardening effect of physical and enhanced dynamic wedges at bladder inhomogeneity using EBT3 film dosimeter. J Cancer Res Ther. 2017; 13(1): 97-101, doi: 10.4103/09731482.206244, indexed in Pubmed: 28508840.

22. Rogers D, Walters B, Kawrakow I. BEAMnrc users manual. Nrc Report Pirs. National Research Council of Canada, Ottawa, Canada 2017.

23. Kakakhel MB. Monte Carlo simulations of dynamic radiotherapy treatments. Doctoral dissertation (Queensland University of Technology 2017). https://eprints.qut.edu. au/50903/1/Muhammad_Kakakhel_Thesis.pdf.

24. Pham TM. Simulation of the transmitted dose in an EPID using a Monte Carlo method. Doctoral dissertation (University of Adelaide 2009). https://digital.library. adelaide.edu.au/dspace/bitstream/2440/52448/1/ Pham2009_MSc.pdf.

25. Konefał A, Bakoniak M, Orlef $A$, et al. Energy spectra in water for the $6 \mathrm{MV} X$-ray therapeutic beam generated by Clinac-2300 linac. Radiat Measure. 2015; 72: 12-22, doi: 10.1016/j.radmeas.2014.11.008. 
26. Sheikh-Bagheri D, Kawrakow I, Walters B, Rogers DW. Monte Carlo simulations: efficiency improvement techniques and statistical considerations Integrating new technologies into the clinic: Monte Carlo and image-guided radiation therapy, Proceedings of the 2006 AAPM Summer School. Medical Physics Publishing, Madison, WI 2006: 71-91.

27. Rogers D, Walters B, Kawrakow I. 2011 DOSXYZnrc users manual. Nrc Report Pirs. National Research Council of Canada, Ottawa, Canada 2017.
28. Chow J, Leung M. A graphical user interface for calculation of 3D dose distribution using Monte Carlo simulations. J Phys: Conference Series. 2008; 102: 012003, doi: 10.1088/1742-6596/102/1/012003.

29. Mahdavi SR, Geraily G, Mostaar A, et al. Dosimetric characteristic of physical wedge versus enhanced dynamic wedge based on Monte Carlo simulations. J Cancer Res Ther. 2017; 13(2): 313-317, doi: 10.4103/09731482.183562, indexed in Pubmed: 28643753. 